\title{
The Role of Ultrasound in Rheumatology
}

\author{
Annamaria lagnocco, MD, Fulvia Ceccarelli, MD, Carlo Perricone, MD, and Guido Valesini, MD
}

\begin{abstract}
Sensitive imaging tools are needed to assess disease activity, to detect joint damage, to monitor treatment response, and to predict disease outcome in rheumatic diseases. For its advantages in costs and risks, and for its validity, reproducibility, and sensitivity to change, ultrasonography (US) is playing an increasing role in the evaluation of joints in patients with inflammatory and degenerative disorders. US has demonstrated to be sensitive in the detection of early bone erosions as well as in the depiction of soft-tissue damage. Semin Ultrasound CT MRI 32:66-73 @ 2011 Elsevier Inc. All rights reserved.
\end{abstract}

$T^{1}$ he need of early suppression of inflammation, to prevent persistent damage, determined the development of sensitive imaging techniques able to detect early signs of disease activity and damage. In this regard, ultrasonography (US) is playing an increasing role in the evaluation of joints with inflammatory and degenerative processes. In the last decade, the use of US, characterized by several advantages, such as limited cost, minimal risk to patient safety, good reproducibility, and sensitivity to change, superseded commonly used imaging modalities, such as plain radiography (X-ray) and even more sophisticated tools, such as computed tomography (CT), in the assessment of patients affected by rheumatic diseases. $^{1}$

Radiographic investigation can document a wide range of changes, according to the stage and severity of the illness. Indeed, in the early stages of rheumatoid arthritis (RA), radiographic findings include soft-tissue shadowing around joints (in the hands and feet), juxta-articular osteopenia, and loss of joint space linked with focal eburnation of articular cartilage. ${ }^{2}$ However, radiography is an insensitive tool for the detection of early bone erosions compared with US. ${ }^{3}$ Osteoarthritis (OA) has been traditionally imaged by the use of conventional radiography: common radiological findings of $\mathrm{OA}$ are represented by joint space narrowing, osteophytes, sclerosis, and deformity. However, this technique has evident limitations in imaging hyaline cartilage and soft tissues, which are frequently involved with disease progression over the years. ${ }^{4}$

Magnetic resonance imaging (MRI) has been recently, and successfully, used in the management of rheumatic diseases.

Reumatologia, Dipartimento di Medicina Interna e Specialità Mediche, Sapienza Università di Roma, Rome, Italy.

Address reprint requests to Annamaria Iagnocco, MD, Cattedra di Reumatologia, Dipartimento di Medicina Interna e Specialità Mediche, Sapienza Università di Roma, Viale del Policlinico 155, Rome 00161, Italy. E-mail: annamaria.iagnocco@uniromal.it
This technique offers several advantages, such as 3-dimensional or tomographic viewing perspective, availability of cross-sectional images of the anatomy in any given plane and, overall, the visualization of all the joints structures, included bone marrow. The use of MRI in patients with RA clearly demonstrates soft-tissue changes of synovium and surrounding tissue as well as erosive bony changes, with an excellent correlation between histomorphologic changes of inflamed joints and the MRI findings. The use of this technique requires enhancement with intravenous gadolinium to demonstrate synovitis. Therefore, a pre- and post-gadolinium enhancement $\mathrm{T}$-weighted scan is performed together with a T2-weighted fat-saturated sequence to permit accurate description of bone edema and erosive changes in RA patients. The Outcome Measures in Rheumatology (OMERACT) group has adopted a semiquantitative system to score bone changes by MRI in RA. ${ }^{5}$ This score system has been shown to be sensitive to change at all stages of the disease, especially in relation to the progression of erosive changes evaluated by standard radiography. Nonetheless, the use of MRI in patients affected with RA showed some limitations, including difficult access to scanners in some centers together with the high costs and limited feasibility for the follow-up.

CT scanning is rarely used in clinical practice to image patients with rheumatic diseases. Because of its accuracy in detecting bony erosions in RA patients, it has been used as the gold standard imaging tool for the quantification of bone erosions. However, CT is less accurate than US and MRI in demonstrating soft-tissues changes. ${ }^{6}$ In the recent years, the use of US has been introduced into the daily routine clinical practice in rheumatology. US technique includes grayscale imaging of anatomic structures and blood flow detection with the usage of the Doppler modalities. These are based on the variations of the frequency of a sound beam reflected to the source when it encounters a moving particle. Thus, US became an established imaging technique in the evaluation of 

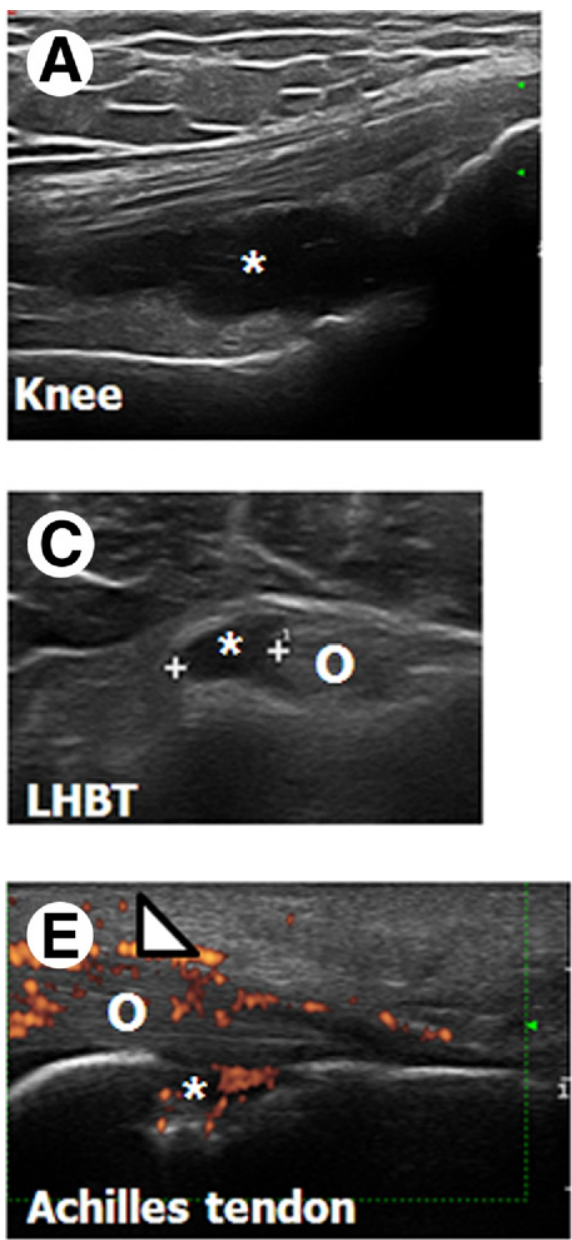
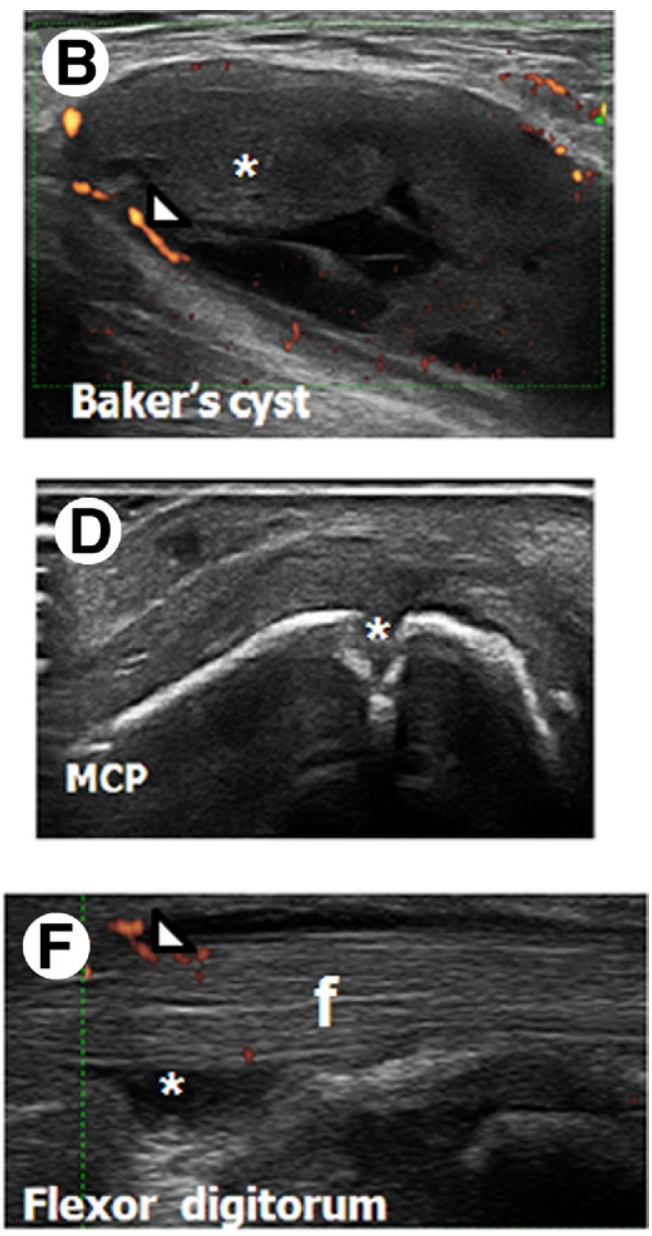

Figure 1 Representative images of OMERACT definitions of US common pathologic findings. From the top left proceeding clockwise. (A) Grayscale anterior para-patellar longitudinal scan of the knee showing the presence of local effusion $\left(^{*}\right)$. (B) Posterior medial longitudinal scan of the knee showing synovial hypertrophy $(*)$ in a Baker's cyst. Note the presence of Doppler signal (arrowhead). (C) Anterior transverse scan in neutral position at the bicipital groove showing the presence of anechoic fluid within the tendon sheath of the long head of the biceps tendon (O) as for tenosynovitis. (D) Dorsal longitudinal scan at the II metacarpal head showing the presence of a bone erosion (*) as an intra-articular discontinuity of the bone surface. (E) Posterior longitudinal scan at the heel showing the presence of a hypoechoic and thickened Achilles tendon $(\mathrm{O})$ at its bony attachment on the calcaneus as for enthesopathy. Note the presence of Doppler signal (arrowhead) and bone erosions $\left(^{*}\right)$. (F) Volar longitudinal scan at the carpal tunnel showing the presence of hypoechoic material $(*)$ within the tendon sheath of the flexor digitorum that shows Doppler signal (arrowhead) as for tenosynovitis. Note: for Figs. 1-4, images were obtained by the use of the MyLab 70 XVision machine (Esaote, Genoa, Italy) equipped with 4-13 and 6-18 MHz probes. (Color version of figure is available online.)

peri- and intra-articular structures, providing an accurate depiction of soft tissue and bony cortex changes at all stages of the disease. Nonetheless, US showed correlation with clinical and laboratory data. ${ }^{7-10}$ In 2005, the OMERACT US group published consensus US definitions for common pathologic lesions observed in patients with inflammatory arthritis. In particular, synovial fluid was defined as an abnormal hypoechoic or anechoic intra-articular material that is displaceable and compressible, and that does not exhibit Doppler signal; synovial hypertrophy as an abnormal hypoechoic intra-articular tissue that is nondisplaceable and poorly compressible and that may exhibit Doppler signal; tenosynovitis as hypoechoic or anechoic thickened tissue with or without fluid within the tendon sheath that is seen in 2 perpendicular planes, and that may exhibit Doppler signal; enthesopathy as an abnormally hypoechoic (loss of normal fibrillar architecture) and/or thickened tendon or ligament at its bony attachment seen in 2 perpendicular planes, that may exhibit Doppler signal and/or bony changes, including enthesophytes, erosions or irregularity; finally, bone erosion as an intraarticular discontinuity of the bone surface that is visible in 2 perpendicular planes. ${ }^{11}$ Representative images are shown in Figure 1.

Comparison studies between US and other imaging techniques, such as MRI, arthroscopy, scintigraphy, and thermography, confirmed the validity of sonography in the detection of synovial inflammation, ${ }^{12}$ which is more specifically and sensitively recognized since the development of Doppler technologies. ${ }^{13,14}$ These techniques, in fact, are able to assess inflammatory activity both in early disease, when pathologic 
changes may be more subtle, and in established disease, when chronic synovial hypertrophy may be present. Similarly to MRI, semiquantitative scoring systems also have been developed for US. The most commonly used ranges from 0 to 3 according to the severity of the alteration, being $0=$ absent, 1 = mild, 2 = moderate, $3=$ severe. This score is usually calculated for each variable analyzed (synovial fluid, synovial hypertrophy, Doppler signal) and has been applied even in the monitoring of response to treatment with encouraging results.

\section{US in Rheumatoid Arthritis}

US can be usefully applied in the assessment and monitoring of RA. As demonstrated by several studies, the sensitivity of traditional methods in the detection of signs of inflammation can be improved by US. Evaluation of the metacarpophalangeal (MCP), proximal interphalangeal (PIP), and metatarsophalangeal (MTP) joints can be particularly useful in patients with early and established disease. ${ }^{9,15,16}$ In presence of synovial thickening, the application of power Doppler (PD) and color Doppler techniques can help in differentiating between active and inactive inflammation. Short-term follow-up studies demonstrated a correlation between disease activity and the degree of synovial inflammation documented in both gray scale and PD modalities. ${ }^{17-20}$ Concerning the US evaluation of bone erosions, in 1999 Backhaus and colleagues ${ }^{9}$ found that this technique can visualize more erosions than radiography in RA. This evidence was further confirmed when MRI or CT was used as the reference method, especially in patients with early disease. ${ }^{15,16,21}$

Several research groups have evaluated the role of US in the monitoring of the response to treatment in RA patients. Filippucci and colleagues ${ }^{22}$ showed significant decrease in joint cavity widening after intra-articular steroid administration in the small joints of the hands and feet of 20 mostly RA patients. These results have been confirmed by Terslev and coworkers ${ }^{23}$ who, in a study of 51 RA patients treated with intra-articular steroids, showed a decrease of $31 \%$ of the pretreatment area of the synovial membrane displayed on grayscale ultrasound. Moreover, color Doppler signal before and after intra-articular steroid treatment was evaluated by Newman, and colleagues who described a decrease in synovial perfusion in the knees of 8 RA patients. ${ }^{24}$ Administration of tumor necrosis factor alpha (TNF- $\alpha$ )-blocking agents results in significant decrease of vascularization evaluated with PDUS. Hau et al ${ }^{25}$ published a study in which they used PD on the MCP joints of 5 RA patients treated with etanercept and demonstrated a significant decrease of the number of color signals per region of interest after only 1 month of follow-up. A reduction in the signs of synovitis by grayscale and Doppler was shown in the hands and wrists of patients with RA treated with infliximab or etanercept. ${ }^{23,26}$ We evaluated the response to etanercept and adalimumab in RA patients, showing that treatment efficacy was followed by improvement of US findings with correlation with disease activity indexes. ${ }^{27,28}$
Few studies confirmed the role of US in determining the prognosis of RA patients. Taylor and coworkers ${ }^{29}$ published data on 24 patients with early RA treated with methotrexate plus either placebo or infliximab showing that changes in the synovial thickening and joint vascularity measured with Doppler technique in the MCP joints at baseline may predict the magnitude of radiographic changes after 2 years. A study by Naredo and colleagues, ${ }^{30}$ in 42 early RA patients starting disease-modifying antirheumatic drugs, showed a positive correlation between time-integrated values of PD parameters and clinical activity, expressed with the Disease Activity Score-28 (DAS28). Furthermore, US showed positive correlation with radiographic progression. Another study by the same group on 278 RA patients treated with TNF- $\alpha$-blocking agents showed that $\mathrm{PD}$ is prognostic for the level of clinical activity and predictor of progression of the total radiographic score. ${ }^{31}$ Brown and coworkers $^{32}$ analyzed a cohort of 102 RA patients with subclinical disease activity and showed that the presence of synovial hypertrophy and PD signal in the MCP joints is predictive of radiographic deterioration. On the basis of the results of the existing studies, it is possible to conclude that PD and, to a smaller extent, gray-scale synovial thickening assessment, may be predictive of radiographic damage in course of RA.

RA patients show a typical polyarticular involvement, whose evaluation may be time-consuming in daily practice. However, there is not enough evidence showing which joints and synovial recesses are best predictors of disease outcome and response to treatment. An increasing number of studies focused on the assessment of the validity of PDUS in the evaluation of inflammatory activity in selected RA joints, Scheel et $\mathrm{al}^{33}$ suggested that in patients affected with RA, US examination can be simplified by focusing on the palmar side of the fingers' joints with a semiquantitative grading of the II-IV MCP and PIP joints ("sum of 3 fingers" method). Naredo and colleagues ${ }^{34}$ evaluated 94 RA patients with PDUS and proposed a 12-joint evaluation method (including bilateral wrists, second and third MCPs, and second and third PIPs of hands and knee joints) that may be a useful tool in the assessment of overall joint inflammatory activity in RA. More recently, 160 patients with active RA starting a biological agent underwent US examination with PD of 128 synovial sites in 44 joints. ${ }^{31}$ By using a semiquantitative grade ranging from 1 to 3 in all the synovial sites, the authors found a highly significant correlation between PDUS score and the selected count and index for synovitis. This result was maintained when only 24 synovial sites at 12 joints were considered (bilateral elbow, wrist, second and third MCP, knee, and ankle joints), with similar significant correlations with DAS28. ${ }^{31}$ More recently, Backhaus and colleagues ${ }^{35}$ proposed a US score based on the evaluation of the wrists, II and III MCPs and PIPs, II and V MTPs joints of the clinically dominant hand and foot. Synovitis and synovial/tenosynovial vascularity are scored semiquantitatively (grade $0-3$ ) by grayscale and PD. The application of this score, named the German US7 score, was a feasible tool in the examination of patients with arthritis in daily rheumatologic practice and it seems to significantly reflect therapeutic response. 


\section{US in \\ Spondyloarthropathies and Polymyalgia Rheumatica}

US was applied not only in the evaluation of RA patients but also in the assessment of other inflammatory diseases, such as spondyloarthropathies (SpA) and polymyalgia rheumatica (PMR).

A growing number of papers investigated the diagnostic potential of US in the assessment of patients with psoriatic arthritis (PsA), supporting its higher sensitivity compared with clinical examination in the evaluation of synovitis, enthesitis, and tenosynovitis. The US findings detected in PsA patients are similar to those found in patients with other inflammatory conditions, such as RA. Information about the activity of the disease can be obtained by the evaluation of the joint effusion, synovial proliferation, synovial hyperemia and modifications of the bone surface.

US is very sensitive in the assessment of tendon involvement by its detection of exudative or proliferative tenosynovitis, loss of "fibrillar" echotexture, and partial or complete tear. The "dactylitis," a common feature of PsA, can be well evaluated with US. It is possible to detect a variable combination of abnormalities, such as tenosynovitis of the finger or toe flexor tendons, synovitis (mainly distal and PIP joints), and diffuse soft-tissue edema. Entheses can be assessed by US in patients affected with SpA. In the early stages of the disease, the entheses and the adjacent structures may show several morphostructural changes as entheseal thickening, hypoechogenicity, and fibrillar separation caused by intratendineous edema, with or without bursitis and different patterns of PD signal distribution. In the late stages, bony cortex changes may be related to the presence of enthesophytes and/or bone erosions. ${ }^{36}$ Recently, Gutierrez et al ${ }^{37}$ conducted an interesting ultrasonographic study to evaluate the incidence of subclinical enthesitis in patients affected with psoriasis without joint involvement. One third of the examined entheses had signs of enthesopathy in gray-scale, $0.9 \%$ entheses showed PD signal. We analyzed the role of US in the assessment of sacroiliac joints, frequently involved in SpA patients, and found encouraging results. ${ }^{38}$ As for RA, US was used in the monitoring of the response to treatment of patients affected with SpA. Several reports showed a correlation between clinical and ultrasonographic findings. ${ }^{39,40}$

US can be used to depict characteristic pathologic findings of shoulders and hips in patients affected with PMR, thus proving helpful in differential diagnosis with other diseases with similar clinical features. Typical findings include glenohumeral joint synovitis, subdeltoid bursitis (Fig. 2), and biceps tendon tenosynovitis in the shoulders and joint synovitis and trochanteric bursitis in the hips. Still, there is the need to standardize the examination and to assess the interobserver agreement in distinguishing lesions typical of PMR. ${ }^{41}$ Cantini and coworkers ${ }^{42}$ performed a case-control study on 57 consecutive patients with PMR and 114 control patients with bilateral shoulder pain and stiffness attributable to other conditions, such as RA, PsA, SpA, OA, fibromyalgia, or con-

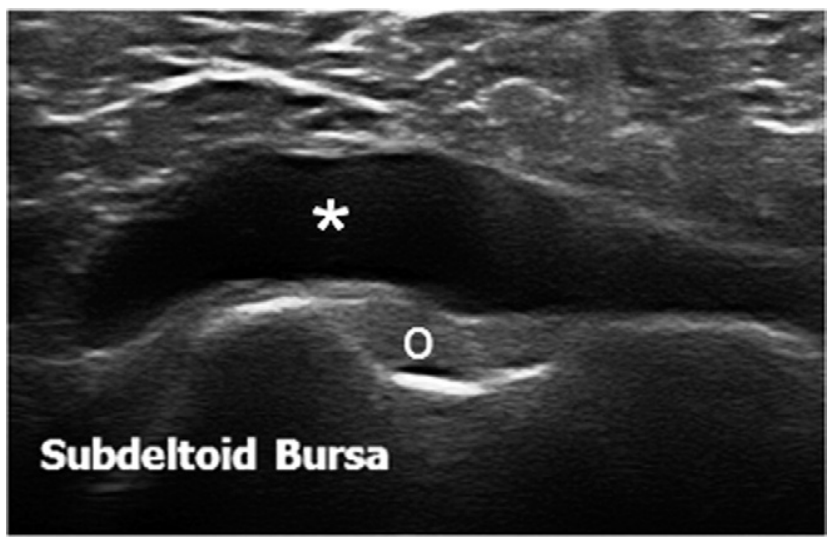

Figure 2 Anterior transverse scan in neutral position at the bicipital groove showing the presence of hypo/anechoic material within the subdeltoid bursa in a patients with PMR as for subdeltoid bursitis surrounding the long head of the biceps tendon $(\mathrm{O})$

nective tissue disease. The authors detected bursitis in all patients, and glenohumeral synovitis and biceps tendon tenosynovitis in $88 \%$ of the cases. An interesting study was conducted by Zaccaria et al, ${ }^{43}$ who included 23 patients with atypical PMR (with normal erythrocyte sedimentation rate), and 88 patients with standard symptomatic PMR. The patients underwent shoulder US scans before receiving steroid therapy and $90 \%$ of them showed signs of bilateral subdeltoid bursitis. This result supports the usefulness of US in patients with clinical signs of PMR but normal erythrocyte sedimentation rate and its help in rendering a diagnosis.

\section{US in Crystal-Related Arthropathies}

US is successfully used in patients affected with crystal-related arthropathies (CA), a group of disorders in which many different species of crystals can be deposited in articular and periarticular tissues, resulting in inflammation and damage. US can detect joint effusion and synovial proliferation, whereas PD can records increased tissue signal, adding information on the inflammatory status of the anatomic structures. Bone erosions, tendinopathy, cartilage involvement, and bursitis are frequently imaged in CA. Nevertheless, there are some ultrasonographic features that could help in the differential diagnosis of CA. Indeed, 3 studies highlighted the main US features of gouty arthritis. Grassi and colleagues ${ }^{44}$ assessed 26 patients with crystal-proven gout and described the spectrum of abnormalities observed. These included the deposition of monosodium urate crystals on the surface of articular cartilage ("double contour" sign), the presence of aggregates of different shapes, echogenicity in the synovial fluid, the deposition of crystals within tendons and the appearance of the tophi. More recently, Thiele and Schlesinger ${ }^{45}$ studied 37 joints of 23 patients affected by gout, observing in 92\% of the patients (and in none of the controls) the irregular band over the superficial margin of the 

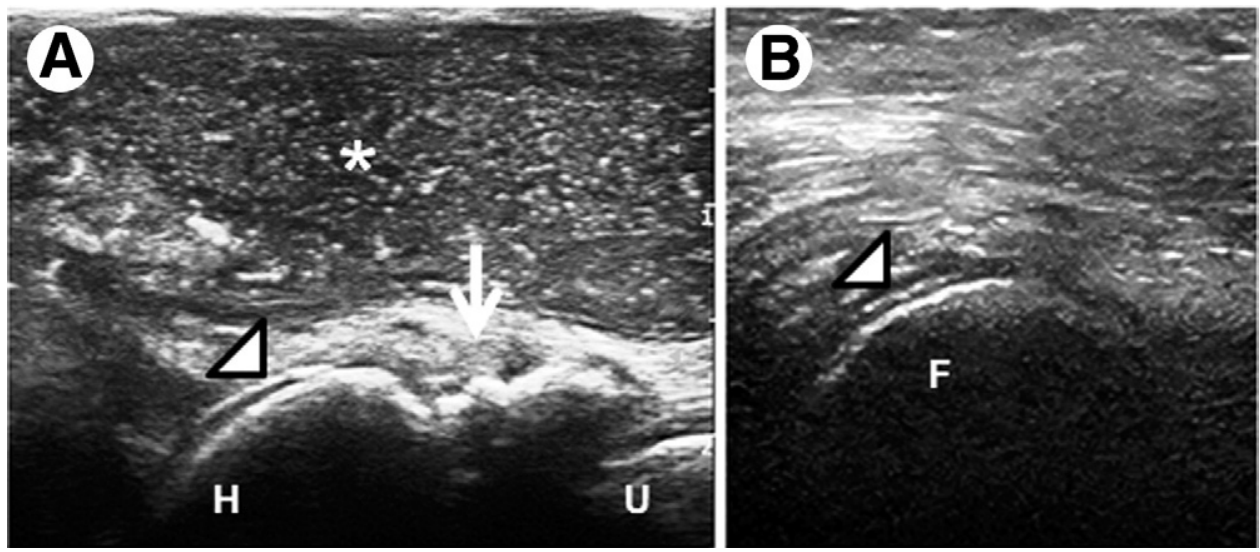

Figure 3 Differential diagnosis between gout and CPPD. (A) Medial longitudinal scan of the elbow showing the deposition of monosodium urate crystals on the surface of the articular cartilage ("double contour" sign) in gout (arrowhead). Note the presence of bone erosions (arrow) below a massive tophus $\left({ }^{*}\right)$ (H, humerus; U, ulna). (B) Posterior lateral longitudinal scan of the knee showing the presence of deposition of pyrophosphate crystals within the femoral hyaline cartilage layer in CPPD (F, femur).

articular cartilage of the metatarsal and metacarpal heads, femoral condyles and humeral head ("double contour" sign).

In all the MCP and MTP joints, tophaceous material was shown together with erosions that were frequently adjacent to the tophaceous material. Furthermore, synovial hypertrophy was imaged as a concentric thickening of the synovial membrane. Interestingly, Wright et $\mathrm{al}^{46}$ found that US is more effective than plain radiographs in the detection of bone erosive damage in the first MTP joint in patients affected by gout. Bone erosions were detected also in joints in which clinical gouty attacks had never occurred. PD has demonstrated increased perfusion in approximately $79 \%$ of the joints from gouty patients. ${ }^{47}$ The decrease of the signal was obtained after colchicine therapy. ${ }^{48}$ US was reliable in the measurement of tophi in chronic gouty patients, showing a good sensitivity to change, associated with urate-lowering therapy, and a good intraobserver correlation at follow-up. ${ }^{49}$

US is effective also in demonstrating calcium pyrophosphate deposits in chondrocalcinosis (CPPD). In 1995, Coari et $\mathrm{al}^{50}$ showed the presence of calcification within condylar cartilage in 43 knees of 28 patients with CPPD, results confirmed by Foldes ${ }^{51}$ and lately by Sofka et al, ${ }^{52}$ who described a case of US-detected meniscal calcification not visualized by conventional radiography. The US criteria for the diagnosis of CPPD deposition were proposed by 2 distinct groups: Grassi et $\mathrm{al}^{44}$ focused their attention on the different pattern of crystal deposition in gout and CPPD disease, showing that the presence of hyperechoic material within the substance of hyaline cartilage is typical of chondrocalcinosis. Frediani et al studied the relation and relationships between the presence of such crystals in articular and periarticular structures and the presence of crystals in the synovial fluids of patients with US evidence of CPPD deposition. They compared US with radiography and found that US was superior in the identification of pyrophosphate crystals. ${ }^{53}$ The Achilles tendon and the plantar fascia of patients with proven (radiographic or microscopic evidence) CPPD disease may show abnormalities in calcifications (detected in 57.9\% of the Achilles ten- dons and in $15.8 \%$ of the plantar fascia) as described by Falsetti et al. ${ }^{54}$ The US features observed included multiple thin linear bands and dishomogeneous rounded hyperechoic deposits. Plantar fascial calcification was always present as a single fine linear echoic band in the superficial region of the insertional tract, apparently not in contiguity with the cortical bone. Interestingly, the authors observed that there were no acoustic shadows behind the calcifications, probably because CPPD crystal deposits in the tendons are unstructured and allow US penetration. ${ }^{55}$ Representative images of comparison between CPPD and gout are shown in Fig. 3.

\section{US in Degenerative Rheumatic Disease}

OA is the most widely studied degenerative disease with US. Indeed, conventional radiography has played an important role in the past in confirming the diagnosis of $\mathrm{OA}$ and in the monitoring of the disease progression. This imaging technique is able to directly visualize bone features of OA, including marginal osteophytes, subchondral sclerosis, and subchondral cysts. However, the assessment of joint space provides only an indirect estimate of cartilage thickness and meniscal integrity. The radiographic definition of $\mathrm{OA}$ is dependent from the evaluation of marginal osteophytes and joint space narrowing. The progression of joint space narrowing is the most commonly used criterion for the assessment of OA outcome and its complete loss, with the boneon-bone contact, is one of the factors considered in the decision for joint replacement. The severity of OA can be estimated with the use of semiquantitative scoring systems. Several grading scales incorporating combinations of features also have been developed, including the most widely used, the Kellgren and Lawrence grade classification. In contrast, the OsteoArthritis Research Society International atlas classification grades separately the tibiofemoral joint space narrowing and osteophytes in each compartment of the knee..$^{55}$ 

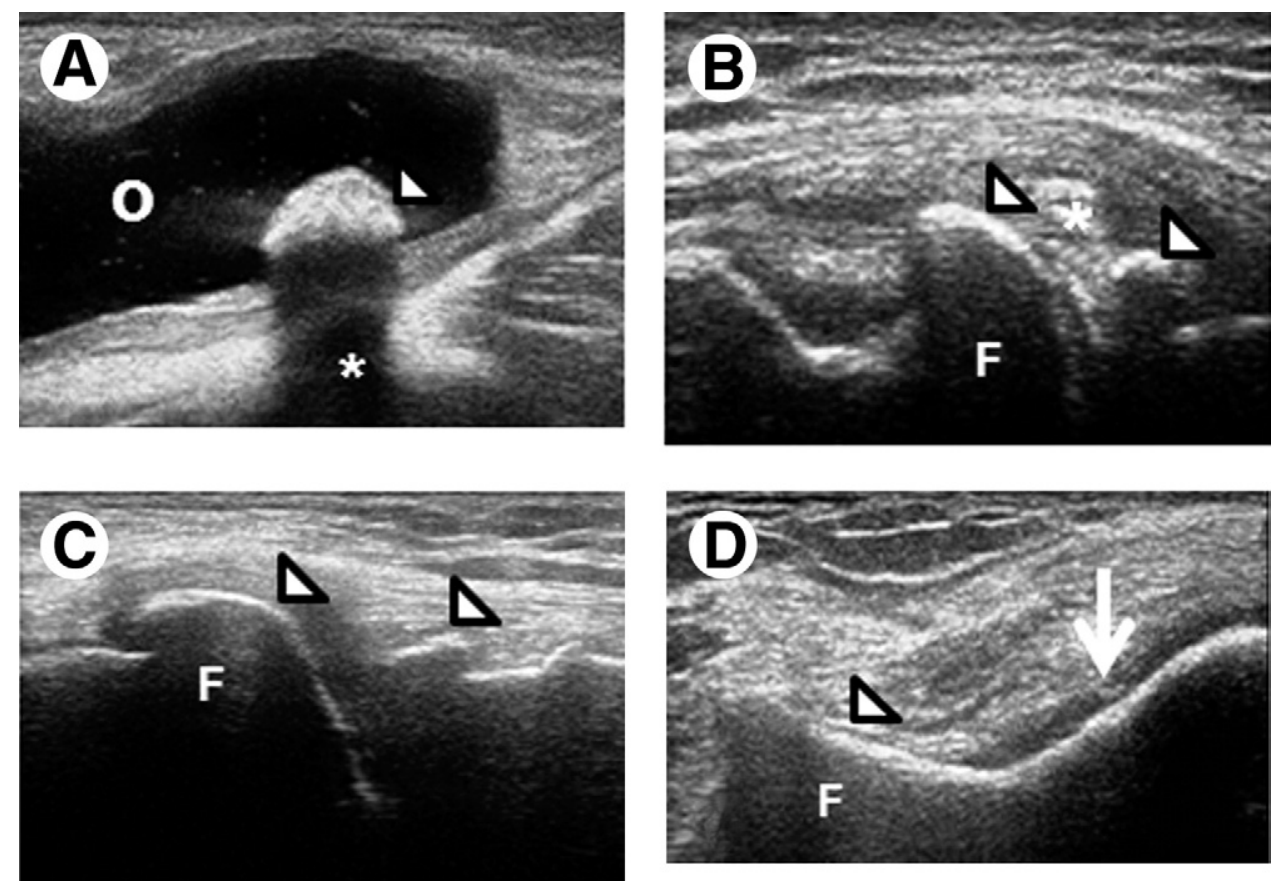

Figure 4 Ultrasonographic features of OA. From upper left proceeding clockwise. (A) Posterior lateral longitudinal scan of the knee showing the presence of a fluctuating calcification (arrowhead) within a Baker's cyst (O). Note the acoustic shadow of the calcification, which was bigger than $10 \mathrm{~mm}\left(^{*}\right)$. (B) Medial longitudinal scan of the knee showing the presence of large osteophytes (arrowheads) visualized both proximal (femoral side) and distal (tibial side) to the joint, together with the presence of calcification of the meniscus $\left(^{*}\right)$ (F, femur; T, tibia). (C) Lateral longitudinal scan of the knee showing the presence of other osteophytes visualized both proximal (femoral side) and distal (tibial side) to the joint (F, femur; $\mathrm{T}$, tibia). (D) Anterior suprapatellar transverse scan of the knee showing the femoral hyaline cartilage (white arrow) abnormalities with evidence of loss of cartilage anechoic echostructure, loss of margins sharpness, irregularities of margins and thinning of the layer till complete disappearance (arrowhead).

$\mathrm{CT}$ is a valuable additional imaging tool when used for the detailed imaging of the osseous changes and the presurgical planning. Besides this, in routine clinical setting, CT plays a minor role as drawbacks of CT are low soft tissue contrast and exposure of patients to ionizing radiation. Nevertheless, $\mathrm{OA}$ affects the whole joint, including cartilage, bone, and intra- and periarticular soft tissues that could be detected only indirectly by radiography and CT. ${ }^{56}$

Although capable of detecting early changes of OA, MRI rarely is used in the routine assessment or initial diagnosis. Compared with radiography, MRI offers several advantages in the imaging of OA being uniquely able to directly depict all anatomic structures of the joint, including the articular cartilage, menisci, intra-articular ligaments, synovium, capsular structures, bone contours, and bone marrow. This allows the evaluation of the joint as a whole organ and provides a more detailed picture of the changes associated with OA. ${ }^{56}$

In recent years US evaluation showed an increasing employment in patients affected by OA, US can detect and evaluate a large number of abnormalities involving hyaline cartilage, synovial fluid and synovial membrane, menisci, joint capsule and bursae as well as the bony cortex. US demonstrates a large set of changes involving the hyaline cartilage from early to late disease. At first, the superficial cartilaginous margins are involved with the formation of the microcleft as the result of tissue deterioration. Then, changes in the echo- texture appear, with the evidence of loss of homogeneity and transparency. Together with disease progression, focal and asymmetric narrowing are usually present and diffuse thinning is charted up to the complete absence of the cartilaginous layer that corresponds to cartilage breakdown and bony denudation (Fig. 4). Cartilaginous changes need to be assessed by correct US scanning technique, based on appropriate patient positioning, adequate probe location, to obtain perpendicular "insonation" of the US beam, and assessment of the controlateral site to perform complete and deep comparison. ${ }^{56}$ Moreover, US can be used to assess bony surface abnormalities. Osteophytes are imaged as cortical protrusions at the joint margin seen in 2 perpendicular planes and visualized as either proximal or distal to the joint. They usually have a posterior acoustic shadow. In erosive hand OA, erosions are imaged as a cortical breakage with a step-down contour defect, seen in 2 planes. The detection of erosions may be difficult because of the interposition of osteophytes, which may determine narrowing of the acoustic window. In a study conducted by our research group, 22 patients with radiographic signs of erosive OA were enrolled, showing that central erosions were detected by US in $72.7 \%$ of patients, with a sensitivity and specificity for the detection of central erosions by US of $73 \%$ and $100 \%$, respectively. ${ }^{57}$

In OA patients with synovitis, joint effusion with synovial thickening and proliferation may appear and can be detected 
by grayscale US. In presence of active inflammation, Doppler modalities are able to demonstrate increased local vascularization within the synovial tissue. OMERACT definitions for synovial fluid and synovial hypertrophy in RA can be applied in OA as well. US is able to identify even minimal joint effusion, which can be either anechoic or inhomogeneously hypoechoic, depending on its composition and on the presence of intra-articular debris and proteinaceous or calcified material. Synovial hypertrophy is another frequent finding in inflamed osteoarthritic joints. In patients with knee OA, US scans over the medial aspect of the joint, frequently demonstrate protrusions of the medial meniscus with displacement of the medial collateral ligaments. To complete US evaluation of osteoarthritic joints, analysis of peri-articular areas, where abnormalities may be occasionally found, is necessary. Typically, those changes consist on the appearance of bursitis that is imaged by US with the representation of abnormal hypo-anechoic, displaceable and compressible intrabursal material. They usually involve the local bursae of the knee, with the appearance of Baker's cysts and anserine bursitis. Also the feet can be affected with the demonstration of bursitis over the medial aspect of the first MTP joint. Several papers showed that US can be applied in the monitoring of the response to treatment with hyaluronic acid in patients affected with OA. ${ }^{58,59}$ Recently, we described the employment of US in the assessment of 53 patients with primary symptomatic knee OA treated for 12 consecutive days with locally applied mud-packs. We observed a significant improvement of the US findings evaluated with a semiquantitative score, that correlated with clinical and laboratory indexes of disease activity. ${ }^{60}$

In conclusion, more work is needed to standardize definitions of pathology and to demonstrate the validity of US. There are no conclusive accepted definitions of sonographic pathology in OA, although those for synovial hypertrophy, synovial effusion, tenosynovitis, enthesitis and erosion developed by the OMERACT have been successfully applied in $\mathrm{OA}$. A number of scoring systems have used in OA and have shown marked heterogeneity. These can be dichotomous, ordinal (based on qualitative, semiquantitative or quantitative domains) or continuous scales. Nonetheless, the study of Doppler or contrast agents in OA is still lacking and there is a paucity of demonstrations on reliability and sensitivity to change for US in OA. ${ }^{61}$

\section{Conclusions}

As widely recognized, US can be considered the new millennium stethoscope of the rheumatologist. Its capacity to be complementary to clinic and to recognize subclinical inflammation makes it a useful instrument that can help the rheumatologist in taking therapeutic decisions and in monitoring response to therapy. The continuous technology improvement with production of high-level equipment together with advances in standardization of the US technique may bring to the future development of new clinimetric-ultrasonographic score indexes, based on the principle of the "physician's intention to treat." Finally, in the next future US may become a useful imaging tool in the prediction of the response to therapy, helping in the choice of the best individual treatment in different rheumatic diseases.

\section{References}

1. Filippucci E, Iagnocco A, Meenagh G, et al: Ultrasound imaging for the rheumatologist. Clin Exp Rheumatol 24:1-5, 2006

2. Fouque-Aubert A, Chapurlat R, Miossec P, et al: A comparative review of the different techniques to assess hand bone damage in rheumatoid arthritis. Joint Bone Spine 77:212-217, 2010

3. Scirè CA, Meenagh G, Filippucci E, et al: Ultrasound imaging for the rheumatologist. XXI. Role of ultrasound imaging in early arthritis. Clin Exp Rheumatol 27:391-394, 2009

4. Meenagh G, Filippucci E, Iagnocco A, et al: Ultrasound imaging for the rheumatologist. VIII. Ultrasound imaging in osteoarthritis. Clin Exp Rheumatol 25:172-175, 2007

5. Østergaard M, Peterfy C, Conaghan P, et al: OMERACT rheumatoid arthritis magnetic resonance imaging Studies. Core set of MRI acquisitions, joint pathology definitions, and the OMERACT RAMRI scoring system. J Rheumatol 30:1385-1386, 2003

6. Riente L, Delle Sedie A, Filippucci E, et al: Ultrasound Imaging for the rheumatologist. XXVII. Sonographic assessment of the knee in patients with rheumatoid arthritis. Clin Exp Rheumatol 28:300-303, 2010

7. Grassi W, Cervini C: Ultrasonography in rheumatology: An evolving technique. Ann Rheum Dis 57:268-270, 1998

8. Wakefield RJ, Brown AK, O'Connor PJ, et al: Musculoskeletal ultrasonography: What is it and should training be compulsory for rheumatologists? Rheumatology 43:821-822, 2004

9. Backhaus M, Kamradt T, Sandrock D, et al: Arthritis of the finger joints: A comprehensive approach comparing conventional radiography, scintigraphy, ultrasound, and contrast-enhanced magnetic resonance imaging. Arthritis Rheum 42:1232-1245, 1999

10. Kane D, Balint PV, Sturrock RD: Ultrasonography is superior to clinical examination in the detection and localization of knee joint effusion in rheumatoid arthritis. J Rheumatol 30:966-971, 2003

11. Wakefield RJ, Balint PV, Szkudlarek M, et al: OMERACT7 Special Interest Group. Musculoskeletal ultrasound including definitions for ultrasonographic pathology. J Rheumatol 32:2485-2487, 2005

12. Karim Z, Wakefield RJ, Quinn M, et al: Validation and reproducibility of ultrasonography in the detection of synovitis in the knee: A comparison with arthroscopy and clinical examination. Arthritis Rheum 50: 387-394, 2004

13. Fiocco U, Cozzi L, Rubaltelli L, et al: Long-term sonographic follow-up of rheumatoid and psoriatic proliferative knee joint synovitis. Br J Rheumatol 35:155-163, 1996

14. Wakefield RJ, Brown AK, O'Connor PJ, et al: Power Doppler sonography: Improving disease activity assessment in inflammatory musculoskeletal disease. Arthritis Rheum 48:285-288, 2003

15. Szkudlarek M, Narvestad E, Klarlund M, et al: Ultrasonography of the metatarsophalangeal joints in rheumatoid arthritis: comparison with magnetic resonance imaging, conventional radiography, and clinical examination. Arthritis Rheum 50:2103-2112, 2004

16. Szkudlarek M, Klarlund M, Narvestad E, et al: Ultrasonography of the metacarpophalangeal and proximal interphalangeal joints in rheumatoid arthritis: A comparison with magnetic resonance imaging, conventional radiography and clinical examination. Arthritis Res Ther R52:8, 2006

17. Backhaus M, Burmester GR, Sandrock D, et al: Prospective two year follow up study comparing novel and conventional imaging procedures in patients with arthritic finger joints. Ann Rheum Dis 61:895-904, 2002

18. Scheel AK, Hermann KG, Ohrndorf S, et al: Prospective 7 year follow up imaging study comparing radiography, ultrasonography, and magnetic resonance imaging in rheumatoid arthritis finger joints. Ann Rheum Dis 65:595-600, 2006

19. Naredo E, Collado P, Cruz A, et al: Longitudinal power Doppler ultrasonographic assessment of joint inflammatory activity in early rheumatoid arthritis: Predictive value in disease activity and radiologic progression. Arthritis Rheum 57:116-124, 2007 
20. Taylor PC, Steuer A, Gruber J, et al: Comparison of ultrasonographic assessment of synovitis and joint vascularity with radiographic evaluation in a randomized, placebo-controlled study of infliximab therapy in early rheumatoid arthritis. Arthritis Rheum 50:1107-1116, 2004

21. Wakefield RJ, Gibbon WW, Conaghan PG, et al: The value of sonography in the detection of bone erosions in patients with rheumatoid arthritis: a comparison with convention radiography. Arthritis Rheum 43:2762-2770, 2000

22. Filippucci E, Farina A, Carotti M, et al: Grey scale and power Doppler sonographic changes induced by intra-articular steroid injection treatment. Ann Rheum Dis 63:740-743, 2004

23. Terslev L, Torp-Pedersen S, Qvistgaard E, et al: Estimation of inflammation by Doppler ultrasound: Quantitative changes after intra-articular treatment in rheumatoid arthritis. Ann Rheum Dis 62:1049-1053, 2003

24. Newman JS, Laing TJ, McCarthy TJ, et al: Power Doppler sonography of synovitis: assessment of therapeutic response-Preliminary observations. Radiology 198:582-584, 1996

25. Hau M, Kneitz C, Tony HP, et al: High resolution ultrasound detects a decrease in pannus vascularisation of small finger joints in patients with rheumatoid arthritis receiving treatment with soluble tumour necrosis factor alpha receptor (etanercept). Ann Rheum Dis 61:55-58, 2002

26. Ribbens $C$, André B, Marcelis $S$, et al: Rheumatoid hand joint synovitis: Gray-scale and power Doppler US quantifications following anti-tumor necrosis factor-alpha treatment: Pilot study. Radiology 229:562-569, 2003

27. Iagnocco A, Filippucci E, Perella C, et al: Clinical and ultrasonographic monitoring of response to adalimumab treatment in rheumatoid arthritis. J Rheumatol 35:35-40, 2008

28. Iagnocco A, Perella C, Naredo E, et al: Etanercept in the treatment of rheumatoid arthritis: Clinical follow-up over one year by ultrasonography. Clin Rheumatol 27:491-496, 2008

29. Taylor PC, Steuer A, Gruber J, et al: Comparison of US assessment of synovitis and joint vascularity with radiographic evaluation in a randomized, placebo-controlled study of infliximab therapy in early rheumatoid arthritis. Arthritis Rheum 50:1107-1116, 2004

30. Naredo E, Collado P, Cruz A, et al: Longitudinal power Doppler ultrasonographic assessment of joint inflammatory activity in early rheumatoid arthritis: Predictive value in disease activity and radiologic progression. Arthritis Rheum 57:116-124, 2007

31. Naredo E, Muller I, Cruz A, et al: Power Doppler ultrasonographic monitoring of response to anti-tumor necrosis factor therapy in patients with rheumatoid arthritis. Arthritis Rheum 58:2248-2256, 2008

32. Brown AK, Conaghan PG, Karim Z, et al: An explanation for the apparent dissociation between clinical remission and continued structural deterioration in rheumatoid arthritis. Arthritis Rheum 58:2958-2967, 2008

33. Scheel AK, Hermann KG, Kahler E, et al: A novel ultrasonographic synovitis scoring system suitable for analyzing finger joint inflammation in rheumatoid arthritis. Arthritis Rheum 52:733-743, 2005

34. Naredo E, Bonilla G, Gamero F, et al: Assessment of inflammatory activity in rheumatoid arthritis: A comparative study of clinical evaluation with grey scale and power Doppler ultrasonography. Ann Rheum Dis 64:375-381, 2005

35. Backhaus M, Ohrndorf S, Kellner H, et al: Evaluation of a novel 7-joint ultrasound score in daily rheumatologic practice: A pilot project. Arthritis Rheum 61:1194-1201, 2009

36. Gutierrez M, Filippucci E, De Angelis R, et al: A sonographic spectrum of psoriatic arthritis: "the five targets." Clin Rheumatol 29:133-142, 2010

37. Gutierrez M, Filippucci E, De Angelis R, et al: Subclinical entheseal involvement in patients with psoriasis: An ultrasound study. Semin Arthritis Rheum 2010 Aug 5 (Epub ahead of print)

38. Spadaro A, Iagnocco A, Baccano G, et al: Sonographic-detected joint effusion compared with physical examination in the assessment of sacroiliac joints in spondyloarthritis. Ann Rheum Dis 68:1559-1563, 2009
39. Fiocco U, Ferro F, Vezzù M, et al: Rheumatoid and psoriatic knee synovitis: Clinical, grey scale, and power Doppler ultrasound assessment of the response to etanercept. Ann Rheum Dis 64:899-905, 2005

40. Unlü E, Pamuk ON, Cakir N: Color and duplex Doppler sonography to detect sacroiliitis and spinal inflammation in ankylosing spondylitis. Can this method reveal response to anti-tumor necrosis factor therapy? J Rheumatol 34:110-116, 2007

41. Scheel AK, Matteson EL, Dasgupta B, et al: Reliability exercise for the polymyalgia rheumatica classification criteria study: The Oranjewoud ultrasound substudy. Int J Rheumatol 2009:738-931, 2009

42. Cantini F, Niccoli L, Nannini C, et al: Inflammatory changes of hip synovial structures in polymyalgia rheumatica. Clin Exp Rheumatol 23:462-468, 2005

43. Zaccaria A, Latinakis G, Oliveri M, et al: The support of the ultrasonography of the shoulder in the diagnosis of polymyalgia rheumatica with normal erythrocyte sedimentation rate. Reumatismo 61:290-297,

44. Grassi W, Meenagh G, Pascual E, et al: "Crystal clear"-sonographic assessment of gout and calcium pyrophosphate deposition disease. Semin Arthritis Rheum 36:197-202, 2006

45. Thiele RG, Schlesinger N: Diagnosis of gout by ultrasound. Rheumatol Oxf 46:1116-1121, 2007

46. Wright SA, Filippucci E, McVeigh C, et al: High-resolution ultrasonography of the first metatarsal phalangeal joint in gout: A controlled study. Ann Rheum Dis 66:859-864, 2007

47. Wright SA, Filippucci E, McVeigh C, et al: Features of gout. A sonographic study. Arthritis Rheum 52:S107-S108, 2005

48. Filippucci E, Ciappetti A, Grassi W: Sonographic monitoring of gout. Reumatismo 55:184-186, 2003

49. Perez-Ruiz F, Naredo E: Imaging modalities and monitoring measures of gout. Curr Opin Rheumatol 19:128-133, 2007

50. Coari G, Iagnocco A, Zoppini A: Chondrocalcinosis: Sonographic study of the knee. Clin Rheumatol 14:511-514, 1995

51. Foldes K: Knee chondrocalcinosis: An ultrasonographic study of the hyaline cartilage. Clin Imaging 26:194-196, 2002

52. Sofka CM, Adler RS, Cordasco FA: Ultrasound diagnosis of chondrocalcinosis in the knee. Skeletal Radiol 31:43-45, 2002

53. Frediani B, Filippou G, Falsetti, et al: Diagnosis of calcium pyrophosphate dehydrate crystal deposition disease: Ultrasonographic criteria proposed. Ann Rheum Dis 64:638-640, 2005

54. Falsetti P, Frediani B, Accai C, et al: Ultrasonographic study of Achilles tendon and plantar fascia in chondrocalcinosis. J Rheumatol 31:22422250, 2004

55. Guermazi A, Burstein D, Conaghan P, et al: Imaging in osteoarthritis. Rheum Dis Clin North Am 34:645-687, 2008

56. Iagnocco A: Imaging the joint in osteoarthritis: A place for ultrasound? Best Pract Res Clin Rheumatol 24:27-38, 2010

57. Iagnocco A, Filippucci E, Ossandon A, et al: High resolution ultrasonography in detection of bone erosions in patients with hand osteoarthritis. J Rheumatol 32:2381-2383, 2005

58. Salini V, De Amicis D, Abate M, et al: Ultrasound-guided hyaluronic acid injection in carpometacarpal osteoarthritis: Short-term results. Int J Immunopathol Pharmacol 22:455-460, 2009

59. Migliore A, Tormenta S, Massafra U, et al: Intra-articular administration of hylan G-F 20 in patients with symptomatic hip osteoarthritis: Tolerability and effectiveness in a large cohort study in clinical practice. Curr Med Res Opin 24:1309-1316, 2008

60. Ceccarelli F, Perricone C, Alessandri Cy, et al: Exploratory data analysis on the effects of nonpharmacological treatment for knee osteoarthritis. Clin Exp Rheumatol 28:250-253, 2010

61. Keen HI, Wakefield RJ, Conaghan PG: A systematic review of ultrasonography in osteoarthritis. Ann Rheum Dis 68:611-619, 2009 